\title{
Messages from Manchester: pilot randomised controlled trial following self-harm
}

Navneet Kapur, David Gunnell, Keith Hawton, Sarmad Nadeem, Samer Khalil, Damien Longson, Rita Jordan, Iain Donaldson, Richard Emsley and Jayne Cooper

\section{Summary}

Studies of therapeutic contact following self-harm have had mixed results. We carried out a pilot randomised controlled trial comparing an intervention (information leaflet listing sources of help, two telephone calls soon after presentation and a series of letters over 12 months) to usual treatment alone in 66 adults presenting with self-harm to two hospitals. We found that our methodology was feasible, recruitment was challenging and repeat self-harm was more common in those who received the intervention (12-month repetition rate $34.4 \% \mathrm{~V}$. $12.5 \%)$.

\section{Declaration of interest}

N.K. chaired the National Institute for Health and Clinical Excellence (NICE) guideline development group and evidence update for the longer-term management of self-harm. N.K., D.G., K.H. are members of the National Suicide Prevention Strategy Advisory Group.
Self-harm is a major health problem internationally and a common cause of presentation to hospital. ${ }^{1}$ It is often repeated and greatly increases the risk of subsequent suicide but the evidence base for effective management is limited. ${ }^{2}$ Low-cost universal interventions that involve contact following self-harm (for example, telephone calls or postcards from health professionals expressing concern) have had varied results. ${ }^{3-6}$ We describe the development and piloting of a similar intervention in Manchester, UK. Our aims were to assess the utility and feasibility of our methodology as well as to obtain a preliminary indication of the impact of our intervention on self-harm repetition.

\section{Method}

We carried out a pilot randomised controlled trial comparing the intervention plus usual treatment with usual treatment alone. Participants were Manchester residents aged over 18 years who presented to two of the three emergency departments in the city with self-harm ('an act of intentional self-injury or poisoning irrespective of the apparent purpose of the act ${ }^{1}$ ) during November 2010 to May 2011. Eligibility was initially determined by clinical teams and information packs were sent out by post. Potential participants were given a telephone number to ring or SMS (text) if they did not wish to be contacted about the study. This consent to approach strategy was approved by the ethics committee as a means of maximising the opportunity for individuals to participate in research. A clinical researcher contacted patients by telephone at least 3 days after presentation inviting them to take part and obtaining informed consent. Individuals for whom the intervention would have been inappropriate or who would be difficult to contact at home soon after hospital attendance were excluded (see online Fig. DS1 for a list of exclusions). Randomisation was conducted via a remote internet-based service (www.sealedenvelope.com).

The intervention was developed using qualitative interviews and focus groups with service users and providers. ${ }^{7}$ It consisted of an information leaflet listing local and national sources of help mailed as soon as possible after consent, two telephone calls within the first 2 weeks, and then a series of letters over a 12-month period (at 1, 2, 4, 6, 8 and 12 months). The calls were made by clinical researchers using a semi-structured format and their purpose was to make contact and facilitate access to appropriate treatment, for example by checking participants had contact details for their primary care or specialist mental health service providers. The format of the letters followed a standard pattern and included a general statement of concern (see online supplement), but were modified where appropriate in response to individual circumstances (for example, 'we are sorry to hear you have been in hospital recently').

Treatment as usual was similar at the two participating sites. A mental health liaison nursing team was in operation 7 days a week between $09.00 \mathrm{~h}$ and $21.00 \mathrm{~h}$ to carry out specialist assessments, with out-of-hours care provided by the duty psychiatrist. Based on available audit data for the study period, in over a third of episodes, patients were referred for mental health follow-up, with one in ten referred to social services or to the voluntary sector.

We investigated the proportion of patients with at least one repeat episode of self-harm resulting in hospital attendance within 12 months, identified from the hospital information systems. In addition, we noted the number of repeat episodes during the same time period. We also investigated use of health services from hospital databases. All outcome data were collected by researchers masked to allocation status. Primary analysis was on an intention-totreat basis and was largely descriptive in nature. We also calculated odds ratios for repetition using logistic regression and incidence rate ratios for number of repeat episodes using negative binomial regression. The study received ethics approval (10/H1014/35) and was registered on the ISRCTN register (ISRCTN65171515).

\section{Results}

Online Fig. DS1 shows participant flows through the trial. In total, 250 potentially eligible participants presented to the study centres and $60 \%(n=150)$ were found to be eligible. Of these, $30 \%$ could not be contacted, and just over a quarter declined, with 66 patients being randomised. Study participants and eligible non-participants were similar in terms of age and gender. Intervention and usual treatment groups were similar in terms of age, gender, and marital status, but those in the intervention group were more likely to be unemployed (69\% v. 59\%), to use methods other than poisoning $(23 \% v .9 \%)$, to have a past history of self-harm $(67 \%$ v. $53 \%)$ and to have had previous psychiatric treatment $(64 \%$ v. $53 \%)$.

Online Table DS1 shows self-harm repetition and resource use in the two groups. The 12-month repeat rate for individuals in the intervention group was $34.4 \%$ v. $12.5 \%$ for the usual treatment group (odds ratio (OR) 3.67, 95\% CI 1.0-13.1, $P=0.046$ ). The 
total number of episodes of repeat self-harm over 12 months was also higher in the intervention group (41 v. 7) (incidence rate ratio (IRR) 5.86, 95\% CI 1.4-24.7, $P=0.016$ ). Adjusting for baseline clinical factors (centre, method of harm (self-poisoning $v$. other), previous self-harm, previous psychiatric treatment), the odds ratio for repetition and incidence rate ratio for number of repeat episodes remained elevated, but in the case of the odds ratio, was no longer statistically significant (repetition: adjusted $\mathrm{OR}=4.35$, 95\% CI 0.9-19.8, $P=0.057$; repeat episodes: adjusted $\mathrm{IRR}=7.16$, 95\% CI 1.6-32.8, $P=0.011)$.

\section{Discussion}

Our intervention was in many ways a composite of contact-type interventions used to date. ${ }^{3-6,8}$ We found that randomising eligible patients, delivering the intervention and determining outcome was feasible although only $60 \%$ of assessed patients met eligibility criteria and we were unable to contact a significant proportion of potential participants. The refusal rate was very similar to previous studies in Western healthcare settings. Our trial was small, but those who received the intervention appeared to be more likely to repeat self-harm than those who received usual treatment alone.

This study had some limitations. It was a pilot investigation in just two centres and we were able to randomise just under half of eligible participants. We included adults resident in Manchester only. We did not record community episodes of self-harm nor consider wider treatment outcomes, ${ }^{9}$ because this was beyond the scope of the pilot. Our findings regarding the effect of the intervention on repetition should be interpreted particularly cautiously since the study was not intended as an efficacy trial. Two much larger randomised trials internationally have suggested that contact interventions could be of benefit in reducing the number of repeat self-harm episodes. ${ }^{4,6}$ A further trial had more equivocal results. ${ }^{5}$ In our study we cannot rule out the possibility that the intervention was associated with a true increase in the risk of repetition. A previous UK intervention trial of a crisis card also found that intervention was associated with increased repetition in some individuals (those who had self-harmed previously). ${ }^{10}$ Our repetition findings could simply reflect the uneven distribution of baseline clinical risk factors between the groups, but adjusting for these made little difference. Our findings might be peculiar to the setting - Manchester has areas of considerable deprivation and some of the highest suicide and self-harm rates in England. It should also be borne in mind that presenting to hospital with repeat episodes could conceivably reflect a reduced threshold for help-seeking or improved engagement with services engendered by receipt of the intervention.

We believe the main implications of our study relate to future research. Studies of these apparently simple contact interventions need to be alert to the possibility of increased repetition and should record adverse effects. Ideally, a range of outcome measures should be collected, perhaps a combination of hospital-determined and self-reported repeat self-harm episodes, as well as indicators of service activity and possibly mental state. Our study illustrates the potential importance of future definitive trials stratifying for clinical variables such as method of harm or previous self-harm at baseline. ${ }^{11}$ Although large samples should help to ensure reasonable balance across groups, lack of stratification has also been an issue with previous research. ${ }^{5}$ Recruiting individuals proved more challenging than anticipated. Potentially eligible participants made up approximately $0.4 \%$ of all emergency department attendances. Future studies that include wider age ranges in sites with fewer out-of-area attendances might recruit more easily. Approaching individuals at the time of hospital attendance and a flexible means of contacting those who are not recruited then, perhaps by telephone, post or SMS, may also increase participation. In future evaluations researchers might also consider the importance of treatment context - interventions could have the greatest impact in settings where the availability of alternative sources of help is limited. Further work is needed to elucidate the active components of therapeutic contact following self-harm before introducing these interventions into routine clinical practice.

Navneet Kapur, MD, FRCPsych, University of Manchester, Centre for Suicide Prevention, Centre for Mental Health and Risk, Manchester; David Gunnell, DSC, FFPHM, School of Social and Community Medicine, University of Bristol, Bristol; Keith Hawton, DSC, FRCPsych, Centre for Suicide Research, The University of Oxford, Department of Psychiatry, Warneford Hospital, Oxford; Sarmad Nadeem, MRCPsych, Samer Khalil, MRCPsych, Damien Longson, FRCPSych, PhD, Rita Jordan, RMN, Iain Donaldson, University of Manchester, Centre for Suicide Prevention, Centre for Mental Health and Risk, Manchester; Richard Emsley, PhD, University of Manchester, Centre for Biostatistics, Institute of Population Health, Manchester; Jayne Cooper, PhD, University of Manchester, Centre for Suicide Prevention, Centre for Mental Health and Risk, Manchester, UK

Correspondence: Nav Kapur, University of Manchester, Centre for Suicide Prevention, Centre for Mental Health and Risk, Jean McFarlane Building, Oxford Road, Manchester M13 9PL. Email: nav.kapur@manchester.ac.uk

First received 16 Jan 2013, final revision 15 Mar 2013, accepted 19 Mar 2013

\section{Funding}

This article presents independent research commissioned by the National Institute for Health Research (NIHR) under its Programme Grants for Applied Research scheme (RP-PG-0606-1247). The views expressed in this publication are those of the authors and not necessarily those of the NHS, NIHR or Department of Health. K.H. and D.G. are NIHR senior investigators. K.H. is also supported by Oxford Health NHS Foundation Trust and N.K. by the Manchester Mental Health and Social Care Trust.

\section{Acknowledgements}

We thank Clive Turpin, Kevin Mackway-Jones Linda Davies Victoria Matthews and Sarah Steeg for their help with the study.

\section{References}

1 Hawton $\mathrm{K}$, Bergen $\mathrm{H}$, Casey $\mathrm{D}$, Simkin S, Palmer B, Cooper J, et al Self-harm in England: a tale of three cities. Soc Psychiatry Psychiatr Epidemiol 2007; 42: $513-21$.

2 National Institute for Clinical Excellence. Longer-Term Care and Treatment of Self-Harm. NICE, 2011.

3 Kapur N, Cooper J, Bennewith O, Gunnell D, Hawton K. Postcards, green cards and telephone calls: therapeutic contact with individuals following self-harm. Br J Psychiatry 2010; 197: 5-7.

4 Carter GL, Clover K, Whyte IM, Dawson AH, D'Este C. Postcards from the EDge: 24-month outcomes of a randomised controlled trial for hospital-treated self-poisoning. Br J Psychiatry 2007; 191: 548-53.

5 Beautrais AL, Gibb SJ, Faulkner A, Fergusson DM, Mulder RT. Postcard intervention for repeat self-harm: randomised controlled trial. Br J Psychiatry 2010; 197: 55-60.

6 Hassanian-Moghaddam H, Sarjami S, Kolahi A-A, Carter GL. Postcards in Persia: randomised controlled trial to reduce suicidal behaviours 12 months after hospital-treated self-poisoning. Br J Psychiatry 2011; 198: 309-16.

7 Cooper J, Hunter C, Owen-Smith A, Gunnell D, Donovan J, Hawton K, et al. "Well, it's like someone at the other end cares about you." A qualitative study exploring the views of users and providers of care of contact-based interventions following self-harm. Gen Hosp Psychiatry 2011; 33: 166-76.

8 Vaiva G, Vaiva G, Ducrocq F, Meyer P, Mathieu D, Philippe A, et al. Effect of telephone contact on further suicide attempts in patients discharged from an emergency department: randomised controlled study. BMJ 2006; 332; 1241-5.

9 Owens C. Interventions for self-harm: are we measuring outcomes in the most appropriate way (letter)? Br J Psychiatry 2010; 197: 502-3.

10 Evans MO, Morgan HG, Hayward A, Gunnell DJ. Crisis telephone consultation for deliberate self-harm patients: effects on repetition. Br J Psychiatry 1999; 175: 23-7.

11 Hatcher S, Owens D. Do get in touch. BMJ 2005; 331: 788-9. 